Proceedings of XIX International Scientific Conference "New Technologies and Achievements in Metallurgy, Material Engineering, Production Engineering and Physics", Częstochowa, Poland, June 7-8, 2018

\title{
The Three and the Four-Electron Interactions in the Penson-Kolb-Hubbard Model
}

\author{
P. TARASEWICZ*
}

Faculty of Pharmacy, Collegium Medicum in Bydgoszcz, Nicolaus Copernicus University in Torun, Jagiellońska 13, 85-067 Bydgoszcz, Poland

An extension of the Penson-Kolb-Hubbard model is investigated. This is made in the zero single-electron hopping integral limit. The extension consists in the addition of the three and the four-electron potentials to the Penson-Kolb-Hubbard Hamiltonian. The pair-hopping term as well as the new ones are treated via the mean field approximation. The ground state properties and the critical temperature are investigated for several cases with different values of the couplings in those new terms. It turns out that the three-electron repulsion as well as the four-electron one lead to the superconductor-Mott insulator transition at some concentration of particles.

DOI: 10.12693/APhysPolA.135.223

PACS/topics: superconductivity, fermion pairs, critical temperature, insulator

\section{Introduction}

The Penson-Kolb model has been known since 1986 [1]. The Penson-Kolb-Hubbard model (PKH) is an extension of this model and recently has attracted much interest due to its potential applicability to problems connected to novel superconductors such as cuprates, iron-based systems, and fullerenes and so on $[2-6]$. This can be justified by the fact that the coherence length of fermion pairs is very short in these materials. In $\mathrm{PKH}$ the intersite pair hopping potential binds electrons or holes into on-site pairs that can move throughout the lattice.

The investigation of the influence of the three and the four fermion interactions on superconductivity due to the local pairing stands for the main motivation of this research. Such terms can be a result of nonlocal interactions of pairs of electrons with phonons. The question of the existence of many-particle interactions such as the four-fermion potential in superconductors has been already asked during last three decades, e.g, in [7-12].

The investigated model is represented by a following Hamiltonian:

$$
H=H_{0}+H^{\prime}
$$

where

$$
\begin{aligned}
& H_{0}=E \sum_{i \sigma} n_{i \sigma}+U \sum_{i} n_{i+} n_{i-}+W \sum_{i} n_{i} n_{i+1} \\
& +U_{3} \sum_{i}\left(n_{i+} n_{i-} n_{i+1}+n_{i} n_{i+1+} n_{i+1-}\right) \\
& \quad+U_{4} \sum_{i} n_{i+} n_{i-} n_{i+1+} n_{i+1-} \\
& \quad-t^{p} \sum_{i}\left(c_{i+}^{*} c_{i-}^{*} c_{i+1-} c_{i+1+}+c_{i+1+}^{*} c_{i+1-}^{*} c_{i-} c_{i+}\right)
\end{aligned}
$$

\footnotetext{
*e-mail: tarasek1@cm.umk.pl
}

and the single-fermion hopping term $H^{\prime}=$ $-t \sum_{i \sigma}\left(c_{i \sigma}^{*} c_{i+1 \sigma}+c_{i+1 \sigma}^{*} c_{i \sigma}\right)$ that is neglected in this paper. Moreover, $n_{i}=\sum_{\sigma} n_{i \sigma}, n_{i \sigma}=c_{i \sigma}^{*} c_{i \sigma}$ while $c_{i \sigma}^{*}$ and $c_{i \sigma}$ are the creation and annihilation operators of a fermion with spin $\sigma= \pm$. Furthermore, $E=E_{0}-\mu$ is the site energy counted with respect to the chemical potential $\mu$. $U, W, U_{3}$ and $U_{4}$ are the coupling constants of the Hubbard, intersite two-fermion, three-fermion and four-fermion interactions, respectively. At last, $-t$ and $-t^{p}$ are the single-fermion and the pair nearest-neighbor hopping integrals.

Now it is useful to apply the pseudospin operators $\rho_{i}^{z}=\frac{1}{2}\left(n_{i}-1\right)$ and $\rho_{i}^{+}=\left(\rho_{i}^{-}\right)^{*}=c_{i+}^{*} c_{i-}^{*}$. Next, the Hamiltonian $H_{0}$ is expressed in terms of these operators and approximated by making use of the mean field method. This procedure gives the following mean field Hamiltonian:

$$
\bar{H}_{0}=\sum_{i} \bar{H}_{i 0}+C L
$$

where $\bar{H}_{i 0}=\tilde{E} \rho_{i}^{z}+\tilde{U}\left(\rho_{i}^{z}\right)^{2}-\Delta\left(\rho_{i}^{+}+\rho_{i}^{-}\right) . \quad z$ stands for the coordination number, $L$ is the number of all lattice sites whereas new parameters are as follows: $\tilde{E}=$ $2 E+U+4 W+2 U_{3}+z m\left(4 W+4 U_{3}+U_{4}\right)+2 z \eta\left(U_{4}+2 U_{3}\right)$, $\tilde{U}=2\left(U+2 U_{3}+z D U_{4}+z(n-1) U_{3}\right)$ and $C=E+W+$ $\frac{\Delta^{2}}{z t^{p}}-2 z U_{4} \eta^{2}-\frac{1}{2} z m^{2}\left(U_{4}+4 U_{3}+4 W\right)-2\left(U_{4}+2 U_{3}\right) z m \eta$. The gap parameter is defined as $\Delta=z t^{p} \frac{1}{L} \sum_{i}\left\langle\rho_{i}^{+}\right\rangle=$ $z t^{p} \frac{1}{L} \sum_{i}\left\langle\rho_{i}^{-}\right\rangle$while $m=\frac{1}{L} \sum_{i}\left\langle\rho_{i}^{z}\right\rangle$ and $\eta=\frac{1}{L} \sum_{i}\left\langle\left(\rho_{i}^{z}\right)^{2}\right\rangle$. $m$ and $\eta$ can be expressed in terms of the average number of fermions per lattice site $n=\frac{1}{L} \sum_{i}\left\langle n_{i}\right\rangle$ and the average number of double occupancies per lattice site $D=$ $\frac{1}{L} \sum_{i}\left\langle n_{i+} n_{i-}\right\rangle$, i.e., $m=\frac{1}{2}(n-1)$ and $\eta=\frac{1}{2} D-\frac{1}{4}(n-1)$. The averages are taken over the grand canonical ensemble with the mean field Hamiltonian (1.3).

Hamiltonian $\bar{H}_{i 0}$ acts in a space spanned by the following vectors: $|+\rangle_{i}=c_{i+}^{*}|00\rangle_{i},|-\rangle_{i}=c_{i-}^{*}|00\rangle_{i},|00\rangle_{i}$ and $|+-\rangle_{i}=c_{i+}^{*} c_{i-}^{*}|00\rangle_{i}$, where $|00\rangle_{i}$ is the vector for 
Eigenvectors and eigenvalues of $\bar{H}_{i 0}$ on

TABLE I a given lattice site.

\begin{tabular}{c|c}
\hline \hline Eigenvector & Eigenvalue \\
\hline$|+\rangle_{i}$ & 0 \\
$|-\rangle_{i}$ & 0 \\
$|E\rangle_{i}=u|+-\rangle_{i}-v|00\rangle_{i}$ & $\frac{1}{4} \tilde{U}+\varepsilon$ \\
$|G\rangle_{i}=u|00\rangle_{i}+v|+-\rangle_{i}$ & $\frac{1}{4} \tilde{U}-\varepsilon$
\end{tabular}

the empty $i$-th site. The results of the diagonalization of this Hamiltonian are shown in Table I. As can be seen from it there are the coefficients $u$ and $v$ that have their squares equal to $u^{2}=\frac{1}{2}\left(1+\frac{\tilde{E}}{2 \varepsilon}\right)$ and $v^{2}=\frac{1}{2}\left(1-\frac{\tilde{E}}{2 \varepsilon}\right)$ with $\varepsilon=\sqrt{\frac{1}{4} \tilde{E}^{2}+\Delta^{2}}$. At this stage there appears the question concerning the ground state and the identification of the proper eigenvector or eigenvectors in the case of degeneration. Note that the situation is clear for $\tilde{U} \leq 0$. In this case the ground state vector corresponds to the $|G\rangle_{i}$ vector. However, even if $\tilde{U}>0|G\rangle_{i}$ can still be the ground state vector up to the moment when $\tilde{U}$ becomes too large and the singly-occupied states dominate. Now let us assume that $\tilde{U}$ is not so large and the total ground state vector is $|G\rangle=\bigotimes_{i=1}^{L}|G\rangle_{i}$. Then the ground state energy per lattice site is $\frac{E_{G}}{L}=C+\frac{1}{L} \sum_{i}\left(\frac{1}{4} \tilde{U}-\varepsilon\right)$.

\section{The discussion of results}

Now let us deal with the investigation of the ground state properties and the behavior of the critical temperature in the function of the concentration of particles in the system. The system of equations from which the gap parameter $\Delta$, the average number of double occupancies per lattice site and the chemical potential can be calculated is determined from the definitions given in Sect. 1 and reads

$$
\begin{aligned}
& \frac{2}{z t^{p}}=\frac{L(\beta)}{\varepsilon}, \quad D=\frac{n}{2}-\frac{1}{2} K(\beta), \\
& n=1-\frac{\tilde{E}}{2 \varepsilon} L(\beta),
\end{aligned}
$$

where $\frac{1}{k T}$ with the Boltzmann constant $k, L(\beta)=$ $\frac{\mathrm{e}^{-\beta \frac{\tilde{U}}{4}} \sinh \beta \varepsilon}{1+\mathrm{e}^{-\beta \frac{\tilde{U}}{4}} \cosh \beta \varepsilon}, K(\beta)=\frac{\mathrm{e}^{\beta \frac{\tilde{U}}{4}}}{\mathrm{e}^{\beta \frac{\tilde{U}}{4}}+\cosh \beta \varepsilon}$. The equation for $D$ can be transformed to another form, i.e., $D=n-$ $1+\frac{1}{2} \frac{\mathrm{e}^{-\beta \frac{\tilde{U}}{4}} \mathrm{e}^{\beta \frac{\tilde{E}}{2}}}{1+\mathrm{e}^{-\beta \frac{\tilde{U}}{4}} \cosh \beta \varepsilon}$ that can be useful later. If $\frac{\tilde{U}}{4}<\varepsilon$, then in the $\beta \rightarrow \infty$ limit one gets the solutions of the system $(2.1)$, namely $\Delta(n)=\frac{z t^{p}}{2} \sqrt{n(2-n)}$ for $n \leq n^{c}$ while if $n>n^{c}$, then $\Delta(n)=0$. $n^{c}$ is the critical concentration of electrons that depends on the values of $U$, $U_{3}, U_{4}$. The average number of pairs is in turn equal to $D=\frac{n}{2}$ for $n \leq n^{c}$ but for $n>n^{c}$ one has two cases, namely, if $n^{c} \leq 1$, then $D=0$ while if $n^{c}>1$, then $D=n-1$. The chemical potential for arbitrary temperature, in general, can be calculated from the formula $\mu=E_{0}+\frac{1}{2}(n-1)\left[z t^{p}+\frac{z}{2}\left(4 W+2 U_{3}\right)\right]+\frac{z}{2} D\left(U_{4}+2 U_{3}\right)+$
$\frac{1}{2}\left(U+4 W+2 U_{3}\right)$ obtained from (2.1). Note that the dependence on the temperature is present in the function $D$ and if $D$ undergoes a jump the same concerns $\mu$.

Some numerical calculations have been done regarding the ground state properties. The $2 D$ square lattice has been investigated. The parameters used in them are as follows: $z=4, t^{p}=0.05 \mathrm{eV}, W=0, E_{0}=0$ and $U=0.19 \mathrm{eV}$. Four cases have been considered: (A) $U_{3}=U_{4}=0$, (B) $U_{3}=0.01 \mathrm{eV}, U_{4}=0$, (C) $U_{3}=0$,

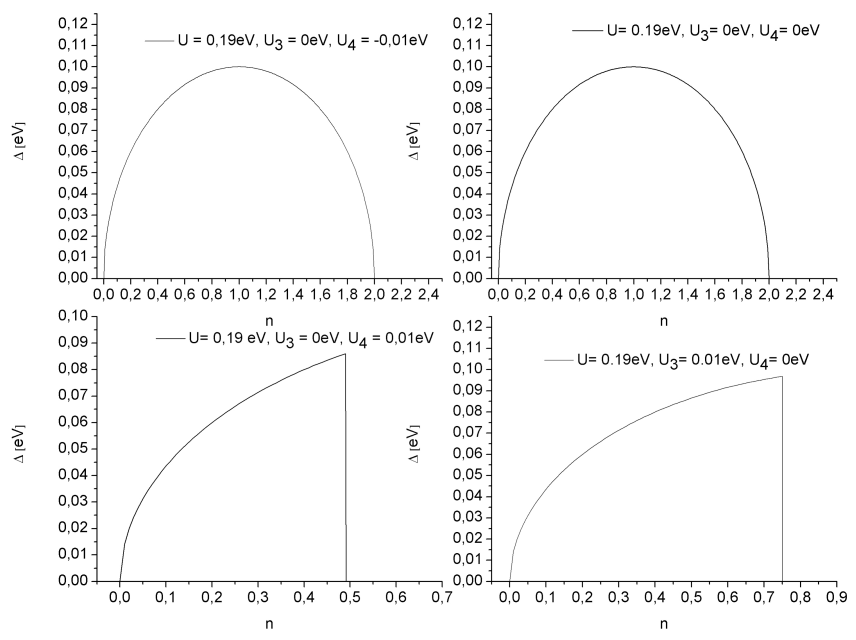

Fig. 1. The dependence of the gap parameter $\Delta$ at $T=0$ on the concentration of electrons $n$ is depicted. The upper right part corresponds to the A case, while the upper left part to the D case. The lower left part corresponds to the $\mathrm{C}$ case, while the lower right one to the B case. Note the first order phase transition to the Mott insulator phase at some $n^{c}$ in parts B and C.
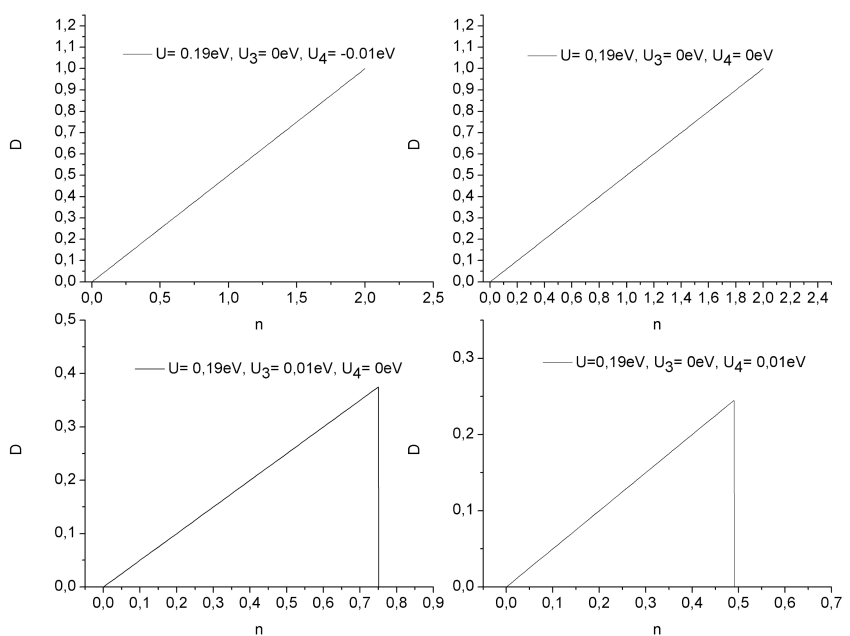

Fig. 2. The dependence of the average number of local pairs per lattice site $D$ at $T=0$ on the concentration of electrons $n$ is depicted. The upper right part corresponds to the A case, while the upper left part to the D case. Note the first order phase transition to the Mott insulator phase at some $n^{c}$ in parts B and C. The lower left part corresponds to the $\mathrm{B}$ case, while the lower right one to the C case. 

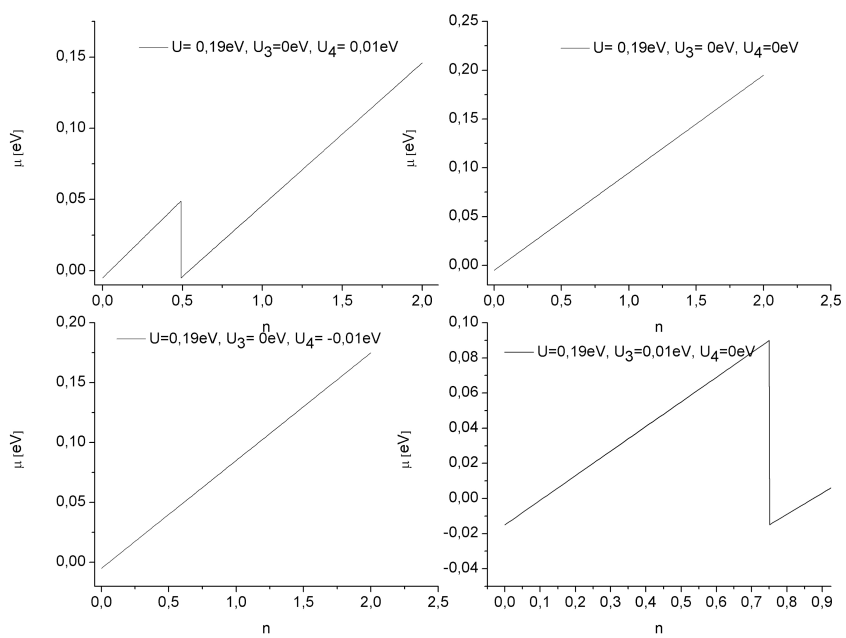

Fig. 3. The dependence of the chemical potential $\mu$ at $T=0$ on the concentration of electrons $n$ is depicted. The upper right part corresponds to the A case, while the upper left part to the $\mathrm{C}$ case. The lower left part corresponds to the $\mathrm{D}$ case, while the lower right one to the B case. Note the first order phase transition to the Mott insulator phase at some $n^{c}$ in parts B and C.
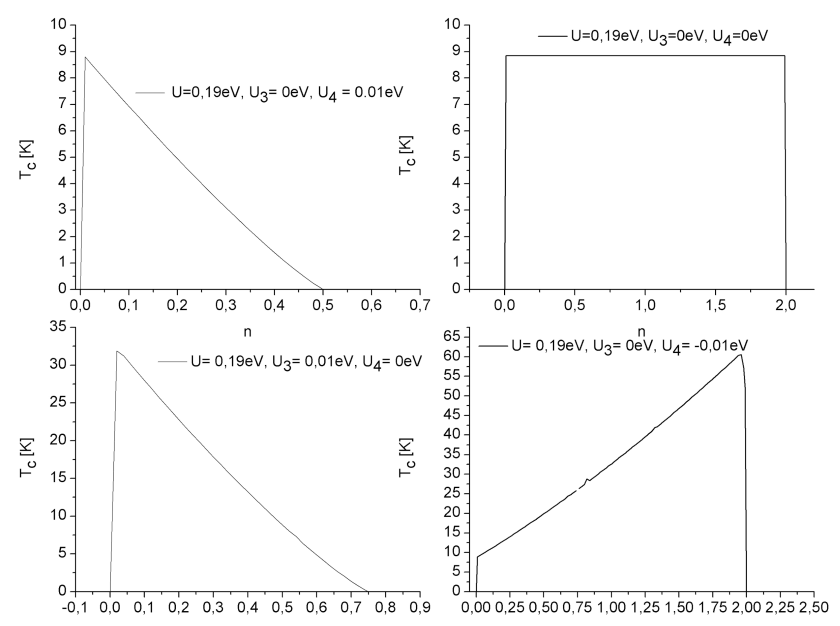

Fig. 4. The dependence of the critical temperature $T_{c}$ on the concentration of electrons $n$ is depicted. The upper right part corresponds to the A case, while the upper left part to the $\mathrm{C}$ case. The lower left part corresponds to the $\mathrm{B}$ case, while the lower right one to the $\mathrm{D}$ case.

$U_{4}=0.01 \mathrm{eV}$, and (D) $U_{3}=0, U_{4}=-0.01 \mathrm{eV}$. All these cases are shown in Figs. 1-3. The dependence of the gap parameter $\Delta$ on $n$ is the same in $\mathrm{A}$ and $\mathrm{D}$ cases and is represented by the curve that has the maximum at half-filling and is symmetric for $n<1$ and $n>1$. This symmetry is destroyed if the three or the four-fermion repulsion appears in the system. One can notice the sharp transition at some $n^{c}$ in both of cases (B and C) which in fact stands for the superconductor-Mott insulator transition. At this point $\Delta$ vanishes and pairs get broken if $n^{c} \leq 1(D=0)$ or some of them survive and are frozen on lattice sites if $n^{c}>1(D=n-1)$ which can be recognized in parts of Figs. 1-3 with cases B and C. The chemical potential $\mu(n)$ has a jump as well in these cases. In the end the dependence of the critical temperature on $n$ is given in Fig. 4. In case $\mathrm{A}$ one observes $T_{c}=0$ for the empty $(n=0)$ and the full band $(n=2)$ but for the rest values of $n$ this is constant, i.e., $T_{c}=8.842 \mathrm{~K}$. However, the situation drastically changes if $U_{3}$ or $U_{4}$ become nonzero. For $U_{3}>0$ and $U_{4}>0$ (B and $\mathrm{C}$ cases) one can see the sharp jump from zero to the maximal value and next the critical temperature gradually drops down to zero at some $n^{c}$. If $U_{4}<0$, then there are two jumps, the first one at $n=0$ and the second one at $n=2$. However, the former is significantly lower than the latter. The symmetry between the $n<1$ case and $n>1$ one is broken.

\section{The final remarks}

In this paper an extended Penson-Kolb-Hubbard model $(\mathrm{PKH})$ has been investigated. The three and the four-fermion potentials have been added to the PKH Hamiltonian and such a system has been handled with the mean field approximation. The dependence of the ground state properties and the critical temperature on the concentration of fermions $n$ have been studied for four cases with different couplings $U_{3}$ and $U_{4}$. The singleelectron hopping has been neglected. It turned out that the both $U_{3}>0$ and $U_{4}>0$ lead to the first order transition at some critical concentration $n^{c}$ that is in fact the superconductor-Mott insulator transition. These facts reveal the serious influence of many-particle interactions on the properties of superconducting systems with local pairing. Further investigations of this problem will be undertaken in the future, e.g., the involvement of the single-electron hopping.

\section{References}

[1] K.A. Penson, M. Kolb, Phys. Rev. B 33, 1663 (1986).

[2] W.R. Czart, S. Robaszkiewicz, Acta Phys. Pol. A 97, 217 (2000).

[3] K.J. Kapcia, S. Robaszkiewicz, R. Micnas, J. Phys. Condens. Matter 24, 215601 (2012).

[4] A. Ptok, K.J. Kapcia, Supercond. Sci. Technol. 28, 045022 (2015).

[5] K.J. Kapcia, W.R. Czart, Acta Phys. Pol. A 130, 617 (2016).

[6] K.J. Kapcia, W.R. Czart, A. Ptok, J. Phys. Soc. Jpn. 85, 044708 (2016).

[7] P. Tarasewicz, D. Baran, Phys. Rev. B 73, 094524 (2006).

[8] P. Tarasewicz, Int. J. Mod. Phys. B 30, 1650124 (2016).

[9] R. Szczęśniak, PloS ONE 7, e31873 (2012).

[10] R. Szczęśniak, A.P. Durajski, A.M. Duda, Ann. Phys. 529, 1600254 (2017).

[11] A.P. Durajski, R. Szczęśniak, Phys. Lett. A 381, 3332 (2017).

[12] R. Szczęśniak, A.P. Durajski, Supercond. Sci. Technol. 27, 125004 (2014). 